\title{
Associations between the IL-6-neutralizing sIL-6R-sgp130 buffer system and coronary artery disease in postmenopausal women
}

\author{
Man Zhou", Wen Dai", Yan Cui, Huan Liu, Yan Li \\ Department of Clinical Laboratory, Wuhan University, Renmin Hospital, Wuhan 430060, China \\ Contributions: (I) Conception and design: M Zhou, W Dai, Y Li; (II) Administrative support: Y Li; (III) Provision of study materials or patients: Y \\ Cui, H Liu; (IV) Collection and assembly of data: M Zhou, W Dai; (V) Data analysis and interpretation: M Zhou, W Dai; (VI) Manuscript writing: \\ All authors; (VII) Final approval of manuscript: All authors. \\ \#These authors contributed equally to this work as co-first author. \\ Correspondence to: Yan Li. Department of Clinical Laboratory, Wuhan University, Renmin Hospital, Jiefang Road 238, Wuhan 430060, China. \\ Email: yanlitf1120@163.com.
}

Background: As a major inflammatory pathway in coronary artery disease (CAD), IL-6 trans-signaling is activated by the IL-6: sIL-6R $\alpha$ binary complex (B) and inhibited by sgp130 through forming the IL6: sIL-6R $\alpha$ : sgp130 ternary complex (T). The aim of the present study was to examine the possible relationship between biomarkers mirroring the IL-6-neutralizing sIL-6R-sgp130 buffer system and CAD in postmenopausal women.

Methods: Our study recruited 155 CAD patients and 181 controls among postmenopausal women. Circulating levels of IL-6, sIL-6R $\alpha$ and sgp130 were detected using an enzyme-linked immunosorbent assay, and the $\mathrm{B} / \mathrm{T}$ ratio was calculated by the specific formulas.

Results: CAD patients showed significantly higher circulating levels of IL-6 and sIL-6R $\alpha$, significantly higher B/T ratio, and significantly lower sgp130 levels than controls (all $\mathrm{P}<0.05)$. Spearman's correlation analysis indicated that IL-6 levels $(\mathrm{r}=0.185, \mathrm{P}<0.01)$ and $\mathrm{B} / \mathrm{T}$ ratio $(\mathrm{r}=0.319, \mathrm{P}<0.01)$ were positively related to Gensini scores, while elevated sgp130 levels were significantly associated with decreased Gensini scores $(\mathrm{r}=-0.565, \mathrm{P}<0.001)$. In addition, multiple regression analysis showed that Gensini scores were negatively associated with serum sgp130 levels $(\beta$-coefficient $=-0.318, \mathrm{P}<0.001)$ and had a positive association with IL-6 levels $(\beta$-coefficient $=0.138, \mathrm{P}<0.05)$. Multivariate logistic regression analysis identified that after adjusting for confounding factors, higher sgp130 remained an independent predictor of lower incidence of $\mathrm{CAD}$ in women after menopause (OR $=0.904 ; 95 \% \mathrm{CI}: 0.837-0.976, \mathrm{P}=0.010)$. Moreover, sgp130 levels at $136.01 \mathrm{ng} / \mathrm{mL}(\mathrm{AUC}=0.957,95 \% \mathrm{CI}: 0.928-0.986, \mathrm{P}<0.001)$ and $\mathrm{B} / \mathrm{T}$ ratio at 1.51 (AUC $=0.765 ; 95 \%$ CI: $0.702-0.828, \mathrm{P}<0.001)$ were effective cut-off points to determine the presence of $\mathrm{CAD}$ based on receiver operating characteristic curves.

Conclusions: Based on this small case-control study, sgp130 and B/T ratio in the IL-6-neutralizing sIL6R-sgp130 buffer system may be promising biomarkers for CAD diagnosis and assessments of coronary stenosis severity in postmenopausal women.

Keywords: Gensini scores; interleukin-6; soluble interleukin-6 receptor $\alpha$; soluble glycoprotein 130; binary/ ternary complex ratio

Submitted Nov 27, 2019. Accepted for publication Jan 03, 2020.

doi: 10.21037/atm.2020.02.27

View this article at: http://dx.doi.org/10.21037/atm.2020.02.27 


\section{Introduction}

Coronary artery disease (CAD) is recognized to be one of the leading causes of death among postmenopausal women due to the lack of estrogen that protects the cardiovascular system (1). Therefore, it is important for women after menopause to understand potential biomarkers, which would greatly contribute to the prevention, diagnosis and treatment of CAD.

Inflammation can induce plaque instability and rupture, which can help trigger and drive atherosclerosis and eventually CAD. Interleukin 6 (IL-6) is an upstream proinflammatory cytokine abundant in human atherosclerotic plaques and has been considered an independent risk factor for CAD $(2,3)$. Circulating IL-6 and the soluble form of the membrane bound alpha IL-6 receptor (sIL-6R $\alpha$ ) can form the heterodimer IL-6: sIL-6R $\alpha$ (hereafter termed "B" for binary complex), which binds directly to the ubiquitously expressed membrane bound beta IL-6 receptor, also called glycoprotein 130 (gp130), and transduces a pro-inflammatory cascade known as the trans-signaling pathway (4).

There is ample evidence to support the potential involvement of IL-6-trans signaling in the pathogenesis of atherosclerosis. A zoological study has shown that hepaticspecific gp130-/- mice are resistant to atherosclerosis, probably owing to macrophage infiltration of atherosclerotic plaques and reduction of acute phase protein levels (5). Another study has identified that the IL-6 trans-signaling pathway plays a critical role in maintaining chronic inflammation in atherosclerosis (6).

Interestingly, the soluble form of gp130 (sgp130) suggests a potential therapeutic paradigm because it selectively inhibits the pro-inflammatory IL-6 trans-signaling pathway, through competitively binding to B with gp130, forming the ternary complex IL-6: sIL-6R $\alpha$ : sgp130 (hereafter termed “T”) (7). A specific antagonist (sgp130Fc) constructed by dimerizing two extracellular domains of sgp130 with a human IgG1-Fc has been developed recently (8). An animal study has demonstrated that low density lipoprotein (LDL)/- mice treated with sgp130Fc attenuate atherosclerotic lesion progression by decreasing endothelial activation and monocyte recruitment, indicating a protective effect of sgp130 on atherosclerosis (9).

To sum up, IL-6 trans-signaling is tightly regulated by active $B$ and inactive T. Because IL- 6 cannot function without its two soluble receptors (and vice versa), we managed to assess the concentrations of the two functional complexes by determining serum IL-6, sIL-6R $\alpha$ and sgp130 levels, and to calculate $\mathrm{B} / \mathrm{T}$ ratio, which can indirectly mirror IL-6 trans-signaling. Then we asked whether these parameters of the IL-6-neutralizing sIL-6R-sgp130 buffer system can predict the risk and severity of CAD in postmenopausal women.

\section{Methods}

\section{Study population}

A total of 336 postmenopausal women who had symptoms of chest tightness and pain were recruited from December 2018 to September 2019 to the Department of Cardiology at Renmin Hospital of Wuhan University. As determined by coronary angiography, individuals with more than $50 \%$ stenotic lesions in at least one of the 15 coronary segments in the three major coronary arteries were diagnosed with CAD (10). While control subjects showed less than $50 \%$ occlusion in all coronary arteries defined as free of significant stenosis, or completely normal coronary arteries (11). All subjects receiving thrombolytic therapy and hypolipidemic drugs, or those with other severe diseases, such as tumor, infection, autoimmune disease, hematopathy, chronic hepatic dysfunction and acute kidney injury, were excluded. Based on these inclusion and exclusion criteria, $155 \mathrm{CAD}$ patients and 181 controls were enrolled.

This study was approved by the Medical Ethics Review Committee of Renmin Hospital, Wuhan University, China. All participants signed written informed consent in conformity to policies of the Renmin Hospital of Wuhan University Ethics Committee.

\section{Quantitative assessment of the severity of coronary artery stenosis}

The severity of CAD was quantitatively evaluated based on Gensini scores according to coronary angiography results, including lesion location, degree of stenosis and numbers of vascular stenosis. Stenosis location was scored as follows: 5 points for the left main coronary artery (LM), 2.5 points for the proximal left anterior descending artery (LAD) or proximal left circumflex artery (LCX), 1.5 points for the mid LAD, and 1 point for the distal LAD and mid or distal LCX. Severity of stenosis was scored as follows: 1 point for $<25 \%$ stenosis, 2 points for $26-50 \%$ stenosis, 4 points for $51-75 \%$ stenosis, 8 points for $76-90 \%$ stenosis, 16 points for $91-99 \%$ stenosis, and 32 points for total occlusion. Each lesion was given a score calculated by multiplying the lesion 
location by severity of stenosis. The total score for each patient was the sum of all lesion scores $(12,13)$.

\section{Definition of risk factors}

Hypertension was diagnosed based on the use of antihypertensive drugs or the measurements of systolic/ diastolic blood pressure $\geq 140 / 90 \mathrm{mmHg}$. Type 2 diabetes mellitus was defined as the use of insulin or oral hypoglycemic drugs, or a fasting glucose level $\geq 126 \mathrm{mg} / \mathrm{dL}$ with symptoms of hyperglycemia. Hyperlipidemia was diagnosed based on fasting total cholesterol concentration $\geq 200 \mathrm{mg} / \mathrm{dL}$, triglyceride concentration $\geq 150 \mathrm{mg} / \mathrm{dL}$ or the use of lipid-lowering agents. Hyperuricemia was defined as a fasting uric acid level $\geq 360 \mu \mathrm{mol} / \mathrm{L}$ (in women) or use of drugs to lower uric acid. Smoking/drinking was defined as current smoking/drinking, or cessation of smoking/drinking within the 6 months prior to study enrollment.

\section{Sample collection}

Before pharmacotherapy, venous blood samples were collected within 24 hours after hospitalization. Samples were collected in the morning after fasting for at least 8 hours. Serum samples were obtained by centrifugation at 3,500 rpm for 5 minutes and stored at $-80{ }^{\circ} \mathrm{C}$ until detection.

\section{Laboratory measurements and calculation of B/T ratio}

Serum levels of alanine aminotransferase (ALT), aspartate aminotransferase (AST), creatinine (Cr), uric acid (UA), glucose (Glu), total cholesterol (TC), triglyceride (TG), high-density lipoprotein cholesterol (HDL-C), low-density lipoprotein cholesterol (LDL-C), small dense low-density lipoprotein cholesterol (sdLDL-C) and high-sensitivity C reactive protein (hs-CRP) were determined using a Siemens ADVIA 2400 automatic biochemistry analyzer (Erlangen, Germany). White blood cell (WBC) and red blood cell (RBC) counts were determined using the SysmexXN-20 system (Kobe, Japan). Fibrinogen (FIB) and D-dimer were detected using the SysmexCA-7000 system (Kobe, Japan).

Serum levels of human IL-6, sIL-6R $\alpha$ and sgp130 were measured using commercial enzyme-linked immunosorbent assay (ELISA) kits that were specific for human antigens (Quantikine DY206, DY227, DY228, and DY008; R\&D Systems, Minneapolis, MN, USA) (14). Serum samples were undiluted to measure IL- 6 and diluted 1:100 to measure sIL-6R $\alpha$ or sgp130. All experiments were carried out in accordance with the manufacturer's instructions. The concentrations of B (IL-6: sIL-6R $\alpha$ ) and T (IL-6: sIL-6R $\alpha$ : sgp130) were estimated using the formulas originally raised by Müller-Newen and Garbers (15), then the $\mathrm{B} / \mathrm{T}$ ratio was calculated (16).

\section{Statistical analysis}

All statistical analysis was performed using IBM SPSS Statistics 23.0 and Graphpad Prism 6.0. Continuous data with a Gaussian distribution were expressed as mean \pm SEM, while continuous data with a non-Gaussian distribution were presented as median (interquartile range, IQR). The Student's independent $t$-test as well as one-way ANOVA and post hoc test (S-N-K test or Games-Howell test) were used to assess the significance of differences in continuous, normally distributed variables. The MannWhitney $\mathrm{U}$ test was used to assess differences in continuous, skewed variables. Categorical data were presented as percentage (\%) and compared using the chi-squared test. The association of IL-6, sIL-6R $\alpha$, sgp130 levels and B/ $\mathrm{T}$ ratio with cardiovascular risk factors and Gensini scores were evaluated using Spearman's correlation analysis. Multivariate linear regression was performed to test the relationship between markers in the IL-6-neutralizing sIL6R-sgp130 buffer system and Gensini scores. IL-6, sIL$6 \mathrm{R}$, sgp130 and $\mathrm{B} / \mathrm{T}$ ratio data were $\log _{10}$-transformed. Potential associations between the measured parameters and the incidence of CAD were analyzed using multivariate logistic regression. Receiver operating characteristic (ROC) curves were used to determine optimum cut-off values for Gensini scores and markers in the IL-6-neutralizing sIL6R-sgp130 buffer system for predicting CAD. Differences were considered statistically significant at $\mathrm{P}$ value $<0.05$.

\section{Results}

\section{Characteristics of the study population}

Baseline characteristics of controls and postmenopausal women with $\mathrm{CAD}$ are summarized in Table 1 . CAD patients had a larger frequency of hypertension $(\mathrm{P}<0.05)$ and higher Gensini scores $(\mathrm{P}<0.001)$ than controls. Age, heart rate and most other cardiovascular risk factors, including smoking, drinking, diabetes mellitus, hyperlipidemia, and hyperuricemia were not significantly different between the groups. With regard to the analysis of lipid parameters, 
Table 1 Baseline characteristics between controls and CAD patients

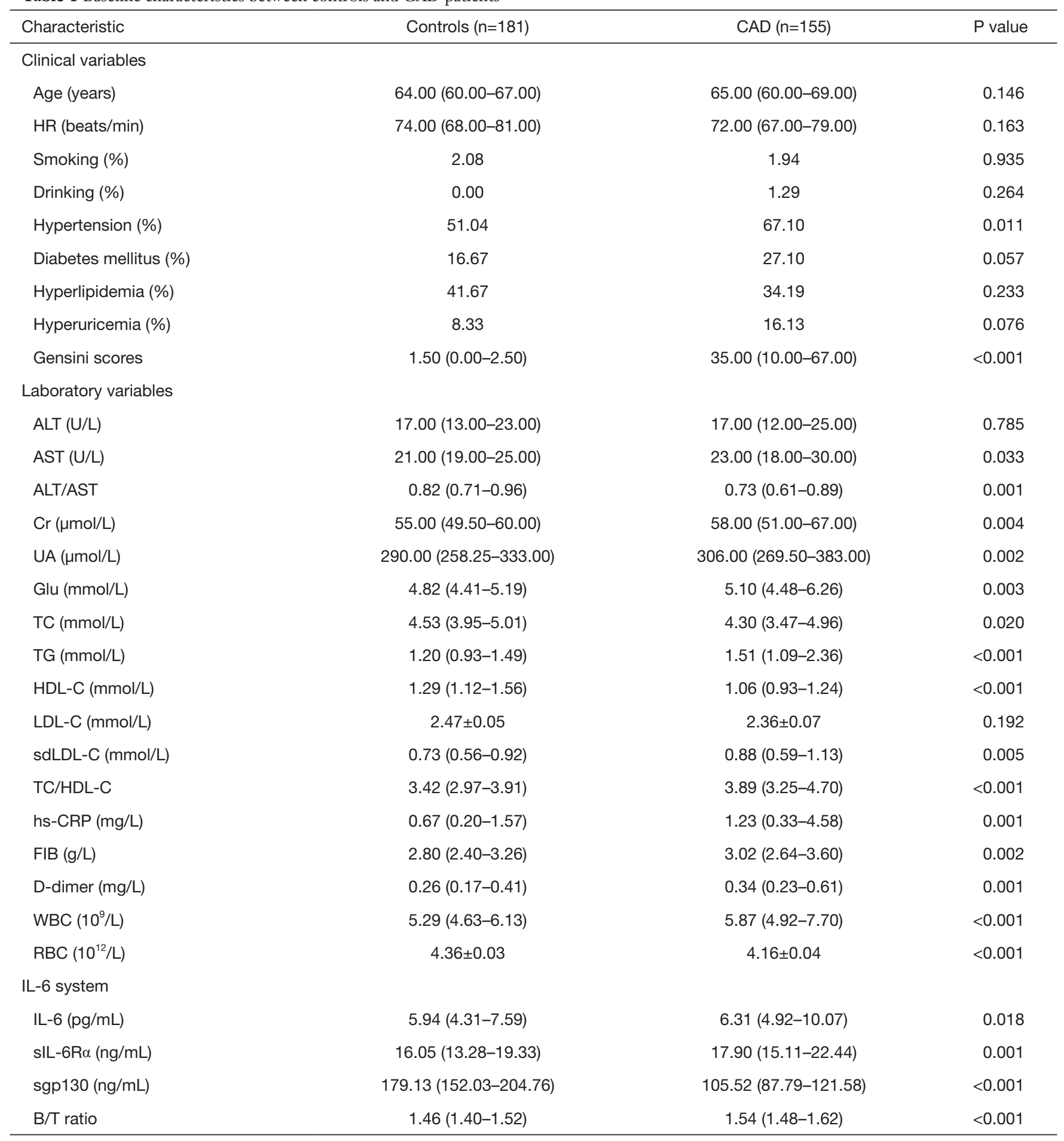

Continuous data were presented as mean \pm SEM or median (interquartile range, IQR) and compared using Student's independent $t$-test or Mann-Whitney $U$ test, while categorical data were presented as percentage (\%) and compared using Chi-Square test. CAD, coronary artery disease; HR, heart rate; ALT, alanine aminotransferase; AST, aspartate aminotransferase; Cr, creatinine; UA, uric acid; Glu, glucose; TC, total cholesterol; TG, triglyceride; HDL-C, high-density lipoprotein cholesterol; LDL-C, low-density lipoprotein cholesterol; sdLDL-C, small dense low-density lipoprotein cholesterol; hs-CRP, high-sensitivity C-reactive protein; FIB, fibrinogen; WBC, white blood cell; RBC, red blood cell; IL-6, interleukin-6; sIL-6R $\alpha$, soluble interleukin-6 receptor $\alpha$; sgp130, soluble glycoprotein 130; B/T ratio, binary/ternary complex ratio. 
Table 2 Correlations of IL-6, sIL-6R, sgp130 levels, and B/T ratio with cardiovascular risk factors in postmenopausal women with CAD

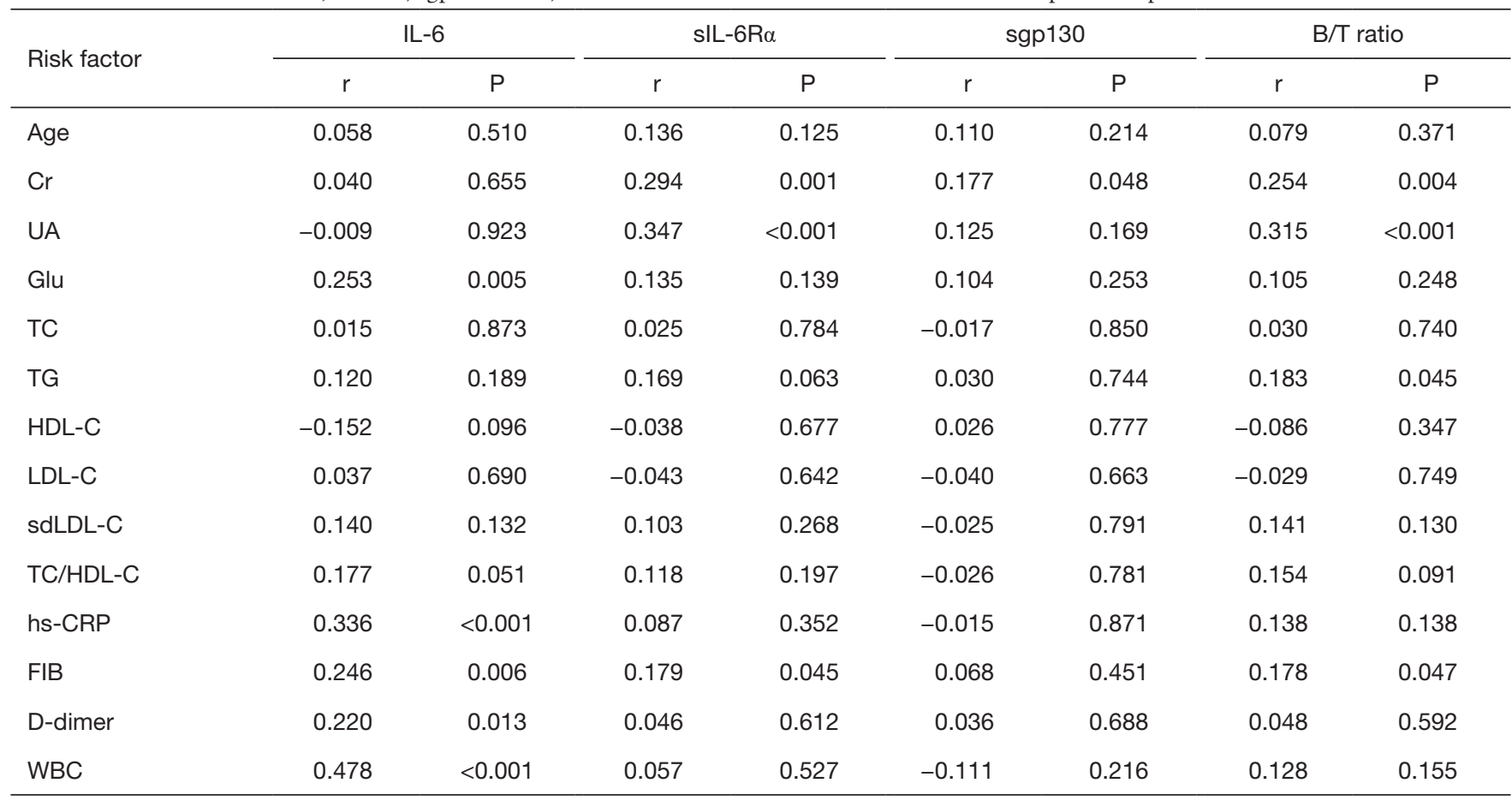

CAD, coronary artery disease; Cr, creatinine; UA, uric acid; Glu, glucose; TC, total cholesterol; TG, triglyceride; HDL-C, high-density lipoprotein cholesterol; LDL-C, low-density lipoprotein cholesterol; sdLDL-C, small dense low-density lipoprotein cholesterol; hs-CRP, high-sensitivity C-reactive protein; FIB, fibrinogen; WBC, white blood cell; IL-6, interleukin-6; slL-6R $\alpha$, soluble interleukin-6 receptor $\alpha$; sgp130, soluble glycoprotein 130; B/T ratio, binary/ternary complex ratio.

CAD patients also had higher levels of unfavorable lipids, such as TG, sdLDL-C and TC/HDL-C, but lower levels of $\mathrm{TC}$ and HDL-C (all $\mathrm{P}<0.05)$. Furthermore, CAD patients showed higher levels of AST, Cr, UA, and Glu (all $\mathrm{P}<0.05$ ), as well as higher levels of WBC, FIB and D-dimer (all $\mathrm{P}<0.01)$. Concerning the IL-6-neutralizing sIL-6R-sgp130 buffer system, serum levels of IL-6 and sIL- $6 \alpha$ as well as the $\mathrm{B} / \mathrm{T}$ ratio were significantly higher in $\mathrm{CAD}$ patients than in controls (all $\mathrm{P}<0.05)$, whereas $\mathrm{CAD}$ patients had lower serum sgp130 levels $(\mathrm{P}<0.001)$.

Correlations of IL-6, sIL-6R, sgp130 levels, and B/T ratio with cardiovascular risk factors in postmenopausal women with CAD

As described in Table 2, high levels of IL-6 had close relations to high levels of hs-CRP $(r=0.336, \mathrm{P}<0.001)$, WBC $(r=0.478$, $\mathrm{P}<0.001)$, FIB $(\mathrm{r}=0.246, \mathrm{P}=0.006)$ and $\mathrm{D}$-dimer $(\mathrm{r}=0.220$, $\mathrm{P}=0.013$ ), indicating a more unfavorable inflammatory reaction and hypercoagulable state. Meanwhile, IL-6 correlated positively with $\mathrm{Glu}(\mathrm{r}=0.253, \mathrm{P}=0.005)$. Moreover,
sIL-6R $\alpha$ showed positive correlations with $\mathrm{Cr}(\mathrm{r}=0.294$, $\mathrm{P}=0.001)$, UA $(\mathrm{r}=0.347, \mathrm{P}<0.001)$, and FIB $(\mathrm{r}=0.179$, $\mathrm{P}=0.045)$. Similarly, $\mathrm{B} / \mathrm{T}$ ratio had positive correlations with the levels of $\mathrm{Cr}(\mathrm{r}=0.254, \mathrm{P}=0.004)$, UA $(\mathrm{r}=0.315, \mathrm{P}<0.001)$, TG $(r=0.183, P=0.045)$ and FIB $(r=0.178, P=0.047)$.

\section{Correlations of circulating levels of IL-6, sIL-6R $\alpha$, and sgp130 as well as B/T ratio with angiographic findings}

According to the coronary angiographic results in Table 3, 89 of the 155 CAD patients had 1-vessel stenosis, 28 had 2 -vessel stenosis and 38 had 3 -vessel stenosis. No statistically significant differences in the IL-6-neutralizing sIL-6R-sgp130 buffer system were detected among the three vessel groups. To further evaluate correlations of IL-6, sIL-6R $\alpha$, sgp130, and B/T ratio with the severity of coronary artery stenosis, Spearman's correlation analysis was performed. As shown in Figure 1, increased Gensini scores were significantly associated with elevated IL-6 levels $(\mathrm{r}=0.185, \mathrm{P}=0.007)$ and $\mathrm{B} / \mathrm{T}$ ratio $(\mathrm{r}=0.319, \mathrm{P}<0.001)$ in postmenopausal women. Moreover, the circulating 
Table 3 Characteristics of the IL-6-neutralizing sIL-6R-sgp130 buffer system in CAD patients stratified by number of stenotic vessels

\begin{tabular}{|c|c|c|c|c|}
\hline Characteristic & 1-vessel $(n=89)$ & 2-vessel $(n=28)$ & 3-vessel $(n=38)$ & $P$ value \\
\hline $\mathrm{slL}-6 \mathrm{R} \alpha(\mathrm{ng} / \mathrm{mL})$ & 17.85 (15.47-22.35) & $18.08(14.84-24.33)$ & $18.38(14.73-22.14)$ & 0.937 \\
\hline sgp130 (ng/mL) & $99.53(85.25-121.14)$ & $108.80(92.61-129.24)$ & 106.39 (94.60-121.19) & 0.432 \\
\hline $\mathrm{B} / \mathrm{T}$ ratio & 1.55 (1.50-1.62) & $1.54(1.47-1.62)$ & $1.55(1.47-1.61)$ & 0.852 \\
\hline
\end{tabular}

Data were presented as median (interquartile range, IQR) and differences among groups were compared using Mann-Whitney $U$ test. CAD, coronary artery disease; IL-6, interleukin-6; sIL-6R $\alpha$, soluble interleukin-6 receptor $\alpha$; sgp130, soluble glycoprotein 130; B/T ratio, binary/ternary complex ratio.
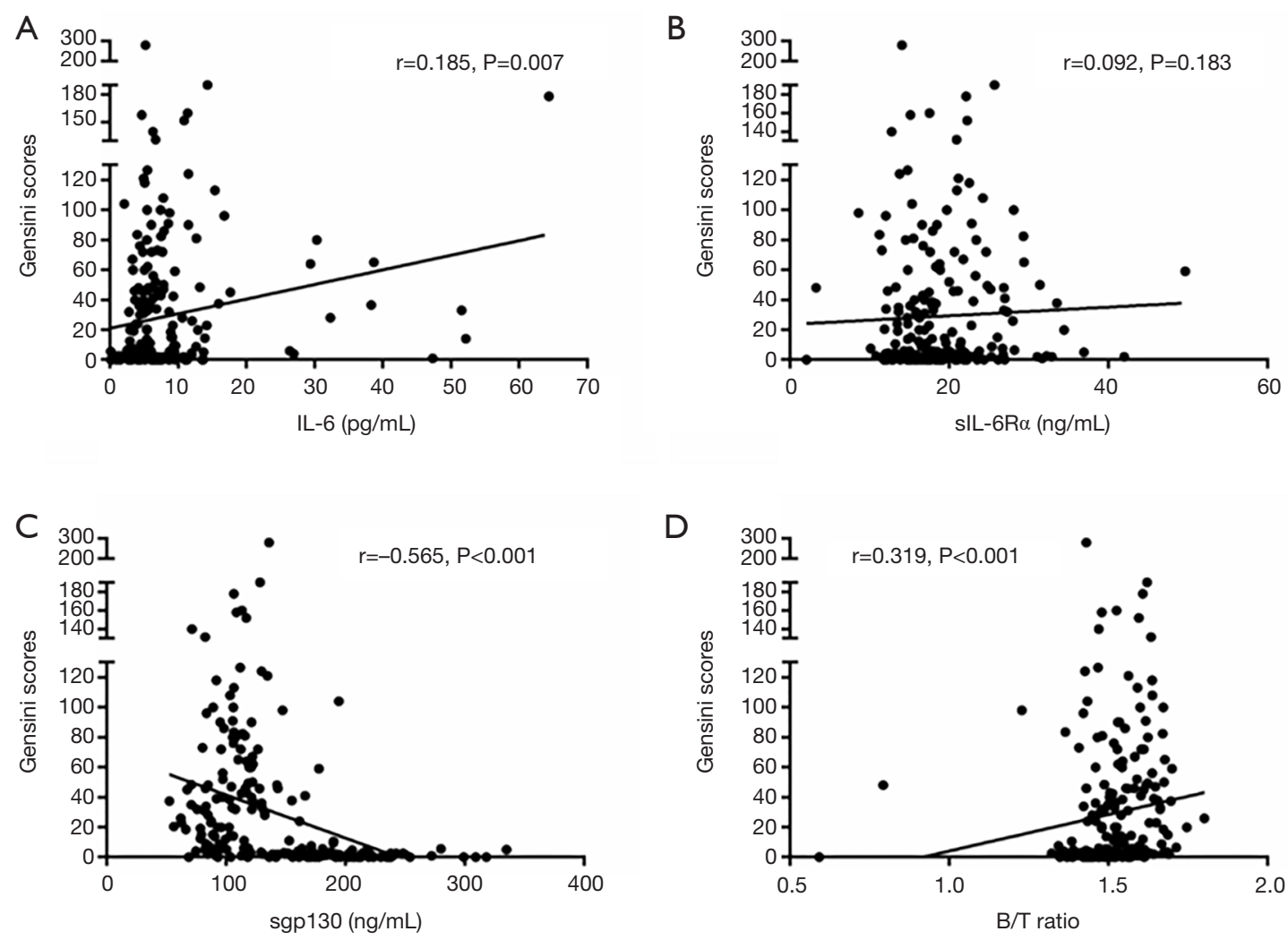

Figure 1 Correlations of IL-6, sIL-6R $\alpha$, sgp130, and B/T ratio with Gensini scores. Scatter diagrams illustrate correlations of circulating levels of IL-6 (A), sIL-6R $\alpha$ (B), sgp130 (C), and B/T ratio (D) with the severity of coronary artery stenosis in postmenopausal women. IL-6, interleukin-6; sIL-6R $\alpha$, soluble interleukin-6 receptor $\alpha$; sgp130, soluble glycoprotein 130; B/T ratio, binary/ternary complex ratio.

levels of sgp130 showed a significant negative correlation with the severity of coronary artery stenosis as defined by Gensini scores $(r=-0.565, \mathrm{P}<0.001)$. In contrast, there was no statistically significant association between sIL-6R $\alpha$ levels and Gensini scores $(\mathrm{r}=0.092, \mathrm{P}=0.183)$. Moreover, multivariate linear regression was performed to identify associations of levels of IL-6, sIL-6R $\alpha$, and sgp130 levels as well as $\mathrm{B} / \mathrm{T}$ ratio with Gensini scores. As summarized in Table 4, in postmenopausal women, Gensini scores were negatively associated with serum sgp130 levels ( $\beta$-coefficient $=-0.318, \mathrm{P}<0.001)$ and had a positive association with IL-6 levels $(\beta$-coefficient $=0.138, \mathrm{P}=0.036)$. 
Table 4 Multivariate linear regression to identify associations of IL-6, sIL-6R $\alpha$, sgp130, and B/T ratio with Gensini scores

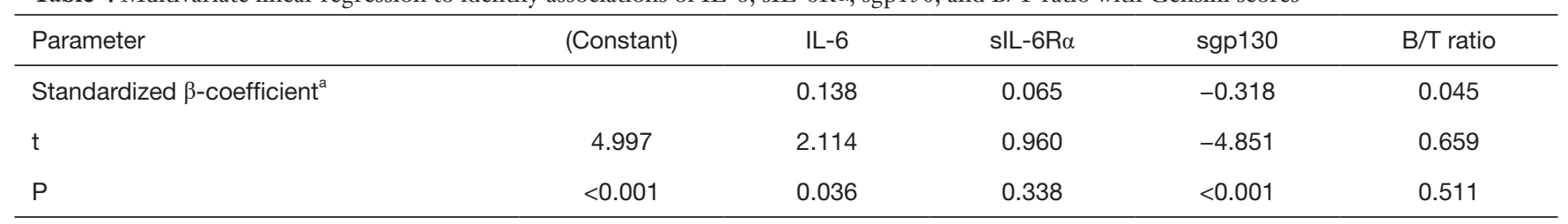

a, dependent variable (Gensini scores). IL-6, interleukin-6; sIL-6R $\alpha$, soluble interleukin-6 receptor $\alpha$; sgp130, soluble glycoprotein 130; B/T ratio, binary/ternary complex ratio.

Table 5 Multivariate logistic regression analysis of Gensini scores and parameters of the IL-6-neutralizing sIL-6R-sgp130 buffer system to identify independent predictors of CAD

\begin{tabular}{lccc}
\hline & & \multicolumn{2}{c}{ Adjusted, OR, P value (95\% Cl) } \\
\hline Parameter & Unadjusted, OR, P value (95\% Cl) & Model 1 & $2.492,0.040(1.043-5.952)$ \\
\cline { 3 - 4 } IL-6 & $1.450,0.016(1.073-1.959)$ & $1.483,0.035(1.029-2.138)$ & $0.927,0.582(0.708-1.214)$ \\
SIL-6R $\alpha$ & $1.035,0.769(0.823-1.301)$ & $1.034,0.821(0.774-1.382)$ & $0.375,0.118(0.110-1.281)$ \\
sgp130 & $0.939,0.719(0.666-1.324)$ & $0.955,0.863(0.568-1.607)$ & $0.904,0.010(0.837-0.976)$ \\
B/T ratio & $0.934,<0.001(0.901-0.967)$ & $0.930,<0.001(0.895-0.965)$ & $1.642^{*} 10^{32}, 0.071\left(0.002-1.504^{*} 10^{67}\right)$ \\
\hline
\end{tabular}

Model 1: after adjustment for age, HR, hypertension, diabetes mellitus, hyperlipidemia and hyperuricemia. Model 2: after adjustment as in Model 1 + Glu, TC, TG, LDL-C, sdLDL-C and hs-CRP. OR, odds ratio; 95\% Cl, 95\% confidence interval; CAD, coronary artery disease; HR, heart rate; Glu, glucose; TC, total cholesterol; TG, triglyceride; LDL-C, low-density lipoprotein cholesterol; sdLDL-C, small dense low-density lipoprotein cholesterol; hs-CRP, high-sensitivity C-reactive protein; IL-6, interleukin-6; slL-6R $\alpha$, soluble interleukin-6 receptor $\alpha$; sgp130, soluble glycoprotein 130; B/T ratio, binary/ternary complex ratio.

\section{Association of Gensini scores and parameters of the $I L-$ 6-neutralizing sIL-6R-sgp130 buffer system with the presence of $C A D$}

To identify factors that may predict the incidence of CAD in postmenopausal women, we performed multivariate logistic regression involving Gensini scores, IL-6, sIL6R $\alpha$, sgp130 and B/T ratio. As shown in Table 5, before adjustment, independent predictors were Gensini scores ( $\mathrm{OR}=1.450 ; 95 \% \mathrm{CI}: 1.073-1.959, \mathrm{P}=0.016)$ and levels of sgp130 (OR =0.934; 95\% CI: 0.901-0.967, $\mathrm{P}<0.001)$. More importantly, these relevancies persisted after adjusting for some conventional risk factors of CAD, such as age, heart rate, hypertension, diabetes mellitus, hyperlipidemia, hyperuricemia, Glu, TC, TG, LDL-C, sdLDL-C and hsCRP. For every unit increase in each covariate, the OR for CAD incidence increased by 2.492 in the case of Gensini scores (95\% CI: 1.043-5.952, $\mathrm{P}=0.040)$ and decreased by 0.904 in the case of sgp130 levels (95\% CI: $0.837-0.976$, $\mathrm{P}=0.010$ ). These results indicate the unfavorable role of Gensini scores and the protective effect of sgp130 on CAD incidence. In contrast, the levels of IL-6, sIL-6R $\alpha$ and $\mathrm{B} / \mathrm{T}$ ratio showed no relationships with the presence of $\mathrm{CAD}$ in any of the three logistic regression models.

\section{ROC curve analysis to assess how well Gensini scores and parameters of the IL-6-neutralizing sIL-6R-sgp130 buffer system can predict $C A D$}

The results of ROC curve analysis of Gensini scores, B/T ratio, and IL-6, sIL-6R $\alpha$ and sgp130 levels to predict CAD in postmenopausal women were shown in Figure 2. Levels of sgp130 were found to have the highest area under the ROC curve (AUC) of 0.957 (95\% CI: 0.928-0.986, $\mathrm{P}<0.001)$. In addition, the most discriminating cut-off value of sgp130 levels was $136.01 \mathrm{ng} / \mathrm{mL}$, which offered a sensitivity of $95.3 \%$ and a specificity of $87.4 \%$. Similarly, using a cut-off value of 5.63 , Gensini scores predicted CAD with a sensitivity of $81.1 \%$ and a specificity of $96.5 \%$ (AUC $=0.937 ; 95 \%$ CI: 0.905-0.969, $\mathrm{P}<0.001$ ), followed by B/T ratio with an AUC of 0.765 (95\% CI: $0.702-0.828$, $\mathrm{P}<0.001)$. However, the AUC values of IL-6 and sIL-6R $\alpha$ 


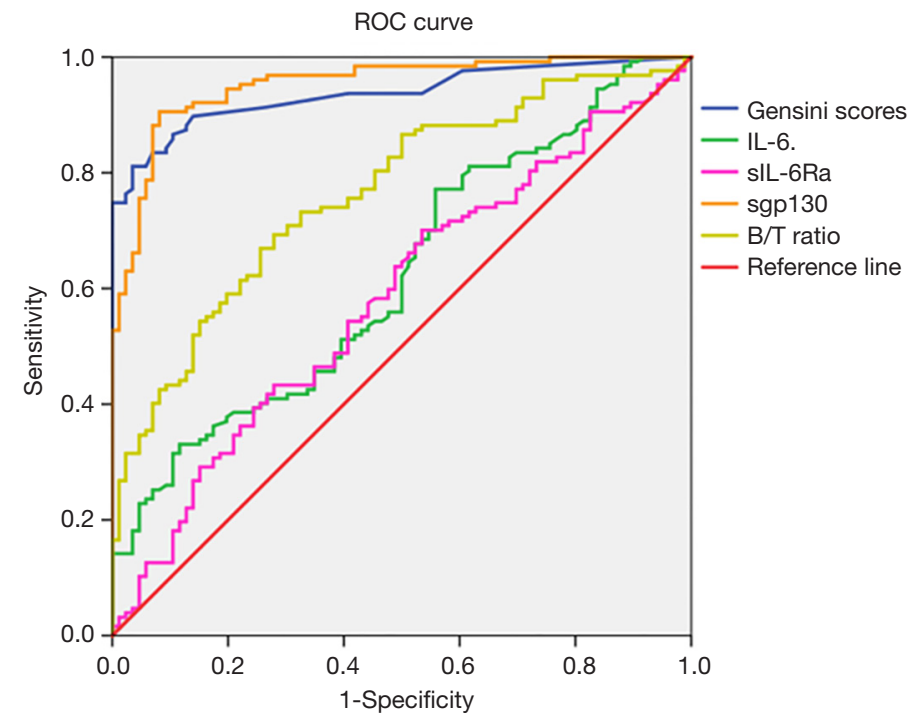

\begin{tabular}{|l|c|c|c|c|c|c|}
\hline Variable & Cut-off value & Sensitivity & Specificity & AUC & $95 \% \mathrm{Cl}$ & P value \\
\hline Gensini scores & $>5.63$ & 0.811 & 0.965 & 0.937 & $0.905-0.969$ & $<0.001$ \\
\hline $\mathrm{IL}-6(\mathrm{pg} / \mathrm{mL})$ & $>8.73$ & 0.331 & 0.884 & 0.622 & $0.547-0.698$ & 0.002 \\
\hline slL-6R $\alpha(\mathrm{ng} / \mathrm{mL})$ & $>16.11$ & 0.701 & 0.465 & 0.583 & $0.506-0.661$ & 0.040 \\
\hline sgp130 $(\mathrm{ng} / \mathrm{mL})$ & $<136.01$ & 0.953 & 0.874 & 0.957 & $0.928-0.986$ & $<0.001$ \\
\hline B/T ratio & $>1.51$ & 0.693 & 0.721 & 0.765 & $0.702-0.828$ & $<0.001$ \\
\hline
\end{tabular}

Figure 2 Receiver operating characteristic (ROC) curve analysis to assess how well Gensini scores and parameters of the IL-6-neutralizing sIL-6R-sgp130 buffer system can predict coronary artery disease in postmenopausal women. AUC, area under the receiver operating characteristic curve; 95\% CI, 95\% confidence interval; IL-6, interleukin-6; sIL-6R $\alpha$, soluble interleukin-6 receptor $\alpha$; sgp130, soluble glycoprotein 130; B/T ratio, binary/ternary complex ratio.

were less than 0.650 .

\section{Discussion}

Cardiovascular events are the most frequent cause of death among women after menopause (17). The present study reports evidence of associations between biomarkers involved in the IL-6-neutralizing sIL-6R-sgp130 buffer system and postmenopausal women with CAD in China. First, the circulating levels of IL-6, sIL-6R $\alpha$, and $\mathrm{B} / \mathrm{T}$ ratio were found to be higher in the CAD population, whereas sgp130 levels were significantly lower. Second, IL-6 levels and $\mathrm{B} / \mathrm{T}$ ratio were positively related to Gensini scores, while elevated sgp130 levels were significantly associated with decreased Gensini scores. Third, Gensini scores and sgp130 levels were identified to be independent predictors of CAD. Fourth, levels of sgp130 had the highest AUC among all markers, with an optimal cut-off value of
$136.01 \mathrm{ng} / \mathrm{mL}$. These findings suggest that it may be possible to predict CAD and severity of coronary stenosis based on straightforward assays of the IL-6-neutralizing sIL-6R-sgp130 buffer system.

Consistent with previous studies $(18,19)$, we found significantly increased circulating IL-6 levels in postmenopausal women with CAD. Moreover, our results indicated that IL-6 levels were positively associated with the severity of coronary artery stenosis. IL-6 is regarded as a pro-atherogenic cytokine. The atherosclerotic lesion size in IL-6-treated apoE-/- mice has been significantly larger than in apoE-/- mice (20), and increased IL-6 levels have been associated with increased risk of cardiovascular events in an observational clinical study (21). As a response to inflammatory stimulation by IL-6, hs-CRP is associated with cardiovascular risk in elderly population (22). Based on our screen of widely used inflammatory biomarkers, we found close associations among the levels of IL-6, hs-CRP, 
FIB, D-dimer and WBC.

In our study, significantly increased levels of sIL-6R $\alpha$ were detected in CAD patients. As a key molecule for IL-6 trans-signaling, sIL-6R $\alpha$ may regulate the effect of IL-6 on endothelial cells, which is crucial for the initiation and development of atherosclerosis (14). Another study has linked increased sIL-6R $\alpha$ levels in myocardium with cardiovascular complications of myocardial infarction, raising the possibility that cardiovascular adverse events may be linked to the induction of IL-6 trans-signaling during cardiac ischemia (23). In addition, Mendelian randomization experiments have indicated that circulating levels of sIL-6R are genetically determined and are causally associated with CAD $(24,25)$.

In our study, decreased circulating levels of sgp130 were related with an increased risk of CAD in postmenopausal women, independently of other conventional risk factors. However, there was no relationship between sgp130 levels and the numbers of diseased vessels. As a controlling factor for IL-6 trans-signaling, sgp130 can modify the progression of IL-6-associated pathology (26). In the literature, the association between sgp130 levels and cardiovascular diseases is controversial. A study of an elderly population with heart failure has shown that increased levels of sgp130 are related to all-cause and cardiovascular mortality (27). Another study has reported that high sgp130 levels predict poor prognosis in patients with a history of myocardial infarction (28). On the other hand, case-control research based on a much larger population has proposed that high sgp130 levels have protective effects against the occurrence of myocardial infarction (29). A serological study has indicated that both patients with CAD in an unstable condition and proportion to advancing CAD have significantly declined levels of endogenous sgp130 and exhibit reinforced IL-6 trans-signaling (9). A recent study has demonstrated that sgp130 levels in patients with unstable or progressive $\mathrm{CAD}$ are significantly lower than in controls and negatively correlate with the number of affected arteries and the severity of coronary atherosclerosis (30). Furthermore, we found that a sgp130 level of $136.01 \mathrm{ng} / \mathrm{mL}$ was an effective cut-off point to predict CAD, suggesting that sgp130 levels could be useful biomarkers for CAD identification. Indeed, endogenous sgp130 levels are influenced by disease genotype and state and vary considerably among different populations (31). Therefore, research on sgp130-based specific inhibition of IL-6 transsignaling in different populations and in different types and stages of disease may guide personalized CAD treatments.
As a specific blocker of IL-6 trans-signaling, sgp130Fc is already in phase I clinical trials for treating chronic inflammatory diseases (32).

Our results showed that CAD patients had significantly higher $\mathrm{B} / \mathrm{T}$ ratios than controls, and a cut-off ratio of 1.51 was a good diagnosis index of CAD in our study. IL-6induced inflammatory responses appear to be regulated by two soluble proteins: sIL-6R $\alpha$, the agonist of IL-6 trans-signaling; and sgp130, identified as an endogenous antagonist of B (33). These two proteins form a systemic buffer system, in which excess sIL-6R $\alpha$ favors IL-6 transsignaling by forming B, while excess sgp130 inhibits trans signaling by forming $\mathrm{T}$ (34). Thus, the $\mathrm{B} / \mathrm{T}$ ratio may reflect the overall level of inflammatory response (16), with a higher ratio linked to higher risk of first cardiovascular events such as CAD, myocardial infarction and ischemic stroke (15). Moreover, as a promising inflammatory biomarker, the $\mathrm{B} / \mathrm{T}$ ratio can predict cardiovascular events in middle-aged individuals defined as having lowintermediate cardiovascular risk (16).

The type and extent of damage to major coronary arteries are valuable parameters for CAD diagnosis and therapy. Gensini scores based on coronary angiography are simple to determine and widely used to evaluate the complexity and severity of coronary stenosis (35), which is why we applied them to our sample. However, the relationship between the IL-6-neutralizing sIL-6R-sgp130 buffer system and severity of plaque lesions has been less investigated. In the present study, we found that Gensini scores had a positive association with IL-6 levels and were negatively associated with serum sgp130 levels, based on univariate and multivariate linear analysis.

The limitations in our study should also be considered. First, it was a cross-sectional study of a relatively small sample from a single center, which limited our statistical power and eliminated the possibility of subgroup analysis. In addition, we did not perform any follow-up analysis. Our results were obtained with elderly postmenopausal women, so they may not be generalizable to other groups of CAD patients. Therefore, the observed association between CAD and the IL-6-neutralizing sIL-6R-sgp130 buffer system should be verified in a larger, more heterogeneous population before advocate for implementing sgp130 and $\mathrm{B} / \mathrm{T}$ ratio as new biomarkers in clinical practice.

Despite these limitations, our study suggests that sgp130 in the IL-6-neutralizing sIL-6R-sgp130 buffer system may have the potential to be a novel marker for predicting incidence of CAD and assessing the severity of coronary 
stenosis in postmenopausal women.

\section{Acknowledgments}

We thank these participants who willingly and generously provided information and samples.

Funding: This research was supported by the National Natural Science Foundation of China (Grant Numbers: $81772265,81572069)$.

\section{Footnote}

Conflicts of Interest: The authors have no conflicts of interest to declare.

Ethical Statement: The authors are accountable for all aspects of the work in ensuring that questions related to the accuracy or integrity of any part of the work are appropriately investigated and resolved. This study was approved by the Medical Ethics Review Committee of Renmin Hospital, Wuhan University, China. All participants signed written informed consent in conformity to policies of the Renmin Hospital of Wuhan University Ethics Committee.

Open Access Statement: This is an Open Access article distributed in accordance with the Creative Commons Attribution-NonCommercial-NoDerivs 4.0 International License (CC BY-NC-ND 4.0), which permits the noncommercial replication and distribution of the article with the strict proviso that no changes or edits are made and the original work is properly cited (including links to both the formal publication through the relevant DOI and the license). See: https://creativecommons.org/licenses/by-nc-nd/4.0/.

\section{References}

1. Yihua L, Yun J, Dongshen Z. Coronary Artery Disease in Premenopausal and Postmenopausal Women. Int Heart J 2017;58:174-9.

2. Luc G, Bard JM, Juhan-Vague I, et al. C-reactive protein, interleukin-6, and fibrinogen as predictors of coronary heart disease: the PRIME Study. Arterioscler Thromb Vasc Biol 2003;23:1255-61.

3. Schieffer B, Schieffer E, Hilfiker-Kleiner D, et al. Expression of angiotensin II and interleukin 6 in human coronary atherosclerotic plaques: potential implications for inflammation and plaque instability. Circulation
2000;101:1372-8.

4. Morieri ML, Passaro A, Zuliani G. Interleukin-6

"Trans-Signaling" and Ischemic Vascular Disease: The Important Role of Soluble gp130. Mediators Inflamm 2017;2017:1396398.

5. Luchtefeld M, Schunkert H, Stoll M, et al. Signal transducer of inflammation gp130 modulates atherosclerosis in mice and man. J Exp Med 2007;204:1935-44.

6. Hartman J, Frishman WH. Inflammation and atherosclerosis: a review of the role of interleukin-6 in the development of atherosclerosis and the potential for targeted drug therapy. Cardiol Rev 2014;22:147-51.

7. Lamertz L, Rummel F, Polz R, et al. Soluble gp130 prevents interleukin-6 and interleukin-11 cluster signaling but not intracellular autocrine responses. Sci Signal 2018. doi: 10.1126/scisignal.aar7388.

8. Baran P, Hansen S, Waetzig GH, et al. The balance of interleukin (IL)-6, IL-6.soluble IL-6 receptor (sIL-6R), and IL-6.sIL-6R·sgp130 complexes allows simultaneous classic and trans-signaling. J Biol Chem 2018;293:6762-75.

9. Schuett H, Oestreich R, Waetzig GH, et al. Transsignaling of interleukin- 6 crucially contributes to atherosclerosis in mice. Arterioscler Thromb Vasc Biol 2012;32:281-90.

10. Liu H, Dai W, Cui Y, et al. Potential associations of circulating growth differentiation factor- 15 with sex hormones in male patients with coronary artery disease. Biomed Pharmacother 2019;114:108792.

11. Liu H, Lyu Y, Li D, et al. Potential relation between soluble growth differentiation factor-15 and testosterone deficiency in male patients with coronary artery disease. Cardiovasc Diabetol 2019;18:21.

12. Gensini GG. A More Meaningful Scoring System for Determining the Severity of Coronary Heart Disease. Am J Cardiol 1983;51:606.

13. Rampidis GP, Benetos G, Benz DC, et al. A guide for Gensini Score calculation. Atherosclerosis 2019;287:181-3.

14. Cui Y, Dai W, Li Y. Circulating levels of sgp130 and sex hormones in male patients with coronary atherosclerotic disease. Atherosclerosis 2017;266:151-7.

15. Ziegler L, Gajulapuri A, Frumento P, et al. Interleukin 6 trans-signalling and risk of future cardiovascular events. Cardiovasc Res 2019;115:213-21.

16. Ziegler L, Frumento P, Wallen H, et al. The predictive role of interleukin 6 trans-signalling in middle-aged men and women at low-intermediate risk of cardiovascular events. Eur J Prev Cardiol 2020;27:122-9.

17. Savonitto S, Colombo D, Franco N, et al. Age at 
Menopause and Extent of Coronary Artery Disease Among Postmenopausal Women with Acute Coronary Syndromes. Am J Med 2016;129:1205-12.

18. Davison S, Davis SR. New markers for cardiovascular disease risk in women: impact of endogenous estrogen status and exogenous postmenopausal hormone therapy. J Clin Endocrinol Metab 2003;88:2470-8.

19. Güdücü N, Görmüş U, Kutay SS, et al. Endogenous sex hormones and their associations with cardiovascular risk factors in post-menopausal women. J Endocrinol Invest 2013;36:588-92.

20. Huber SA, Sakkinen P, Conze D, et al. Interleukin-6 exacerbates early atherosclerosis in mice. Arterioscler Thromb Vasc Biol 1999;19:2364-7.

21. Ridker PM, Rifai N, Stampfer MJ, et al. Plasma concentration of interleukin-6 and the risk of future myocardial infarction among apparently healthy men. Circulation 2000;101:1767-72.

22. Zuliani G, Morieri ML, Volpato S, et al. Insulin resistance and systemic inflammation, but not metabolic syndrome phenotype, predict 9 years mortality in older adults. Atherosclerosis 2014;235:538-45.

23. Kaminski KA, Kozuch M, Bonda T, et al. Coronary sinus concentrations of interleukin 6 and its soluble receptors are affected by reperfusion and may portend complications in patients with myocardial infarction. Atherosclerosis 2009;206:581-7.

24. Sarwar N, Butterworth AS, Freitag DF, et al. Interleukin-6 receptor pathways in coronary heart disease: a collaborative meta-analysis of 82 studies. Lancet 2012;379:1205-13.

25. Swerdlow DI, Holmes MV, Kuchenbaecker KB, et al. The interleukin-6 receptor as a target for prevention of coronary heart disease: a mendelian randomisation analysis. Lancet 2012;379:1214-24.

26. Rose-John S, Waetzig GH, Scheller J, et al. The IL-6/sIL6R complex as a novel target for therapeutic approaches. Expert Opin Ther Targets 2007;11:613-24.

Cite this article as: Zhou M, Dai W, Cui Y, Liu H, Li Y. Associations between the IL-6-neutralizing sIL-6R-sgp130 buffer system and coronary artery disease in postmenopausal women. Ann Transl Med 2020;8(6):379. doi: 10.21037/ atm.2020.02.27
27. Askevold ET, Nymo S, Ueland T, et al. Soluble glycoprotein 130 predicts fatal outcomes in chronic heart failure: analysis from the Controlled Rosuvastatin Multinational Trial in Heart Failure (CORONA). Circ Heart Fail 2013;6:91-8.

28. Ritschel VN, Seljeflot I, Arnesen H, et al. Circulating Levels of IL-6 Receptor and gp130 and Long-Term Clinical Outcomes in ST-Elevation Myocardial Infarction. J Am Heart Assoc 2016. doi: 10.1161/JAHA.115.003014.

29. Moreno Velásquez I, Golabkesh Z, Källberg H, et al. Circulating levels of interleukin 6 soluble receptor and its natural antagonist, sgp130, and the risk of myocardial infarction. Atherosclerosis 2015;240:477-81.

30. Korotaeva AA, Samoilova EV, Chepurnova DA, et al. Soluble glycoprotein 130 is inversely related to severity of coronary atherosclerosis. Biomarkers 2018;23:527-32.

31. Garbers C, Monhasery N, Aparicio-Siegmund S, et al. The interleukin-6 receptor Asp358Ala single nucleotide polymorphism rs2228145 confers increased proteolytic conversion rates by ADAM proteases. Biochim Biophys Acta 2014;1842:1485-94.

32. Scheller J, Garbers C, Rose-John S. Interleukin-6: from basic biology to selective blockade of pro-inflammatory activities. Semin Immunol 2014;26:2-12.

33. Zuliani G, Galvani M, Maggio M, et al. Plasma soluble gp130 levels are increased in older subjects with metabolic syndrome. The role of insulin resistance. Atherosclerosis 2010;213:319-24.

34. Reeh H, Rudolph N, Billing U, et al. Response to IL-6 trans- and IL-6 classic signalling is determined by the ratio of the IL-6 receptor alpha to gp130 expression: fusing experimental insights and dynamic modelling. Cell Commun Signal 2019;17:46.

35. Xu RX, Li S, Li XL, et al. High-density lipoprotein subfractions in relation with the severity of coronary artery disease: A Gensini score assessment. J Clin Lipidol 2015;9:26-34. 